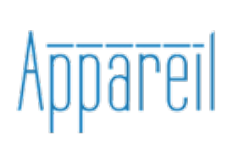

Appareil

$15 \mid 2015$

L'art dans le tout numérique

\title{
Quand la photographie cesse d'en être
}

De l'argentique au numérique

\section{André Rouillé}

\section{(2) OpenEdition}

Journals

Édition électronique

URL : http://journals.openedition.org/appareil/1336

DOI : 10.4000/appareil. 1336

ISSN : 2101-0714

Éditeur

MSH Paris Nord

Référence électronique

André Rouillé, "Quand la photographie cesse d'en être », Appareil [En ligne], 15 | 2015, mis en ligne le 27 octobre 2011, consulté le 01 août 2020. URL : http://journals.openedition.org/appareil/1336 ; DOI : https://doi.org/10.4000/appareil.1336

Ce document a été généré automatiquement le 1 août 2020

\section{(9) $\odot \Theta \Theta$}

Appareil est mis à disposition selon les termes de la Licence Creative Commons Attribution - Pas d'Utilisation Commerciale - Pas de Modification 4.0 International. 


\title{
Quand la photographie cesse d'en être
}

\author{
De l'argentique au numérique
}

André Rouillé

1 La mort récente de Steve Jobs, qui a fait d'Apple la plus brillante et inventive firme d'informatique du monde, a suscité une émotion planétaire. Non pas sur le mode de la ferveur réservée aux vedettes du spectacle, mais sous l'aspect plus mesuré et grave d'un sentiment de gratitude envers l'un des grands acteurs de ce processus immense que vit actuellement l'humanité : l'avènement de l'ère numérique.

2 Sous l'impulsion de Steve Jobs, Apple n'a cessé de proposer des appareils par lesquels l'ère numérique nouvelle est entrée dans la pratique d'un large public. En plaçant les ingénieurs sous l'autorité des designers, en combinant toujours technicité et ergonomie, et en proposant des appareils de rupture, Apple a élargi l'horizon des pratiques et des usages, redéfini les manières d'être et de faire avec le numérique. Et ainsi réussi, en quelque sorte, le tour de force d'ouvrir une époque, c'est-à-dire de faire événement, avec chacun de ses principaux appareils.

3 Le Macintosh, avec ses menus déroulants, ses fenêtres et sa souris, a fait époque dans les années 1980 en faisant entrer dans l'ère numérique les amateurs ignorants de l'informatique. Époque encore avec l'iPod, qui a rendu nomade l'écoute de la musique ; époque aussi avec l'iPhone, qui allie la téléphonie à un ordinateur de poche conçu pour répondre de façon inouie aux multiples besoins quotidiens du citoyen des sociétés contemporaines avancées ; époque enfin avec l'iPad, qui est en train de reconfigurer les positions actuelles des ordinateurs portables, de la presse papier et du livre.

4 Toutes ces époques, véritables révolutions culturelles sectorielles, sont ponctuées par le surgissement d'appareils numériques avec pour enjeux d'inventer à chaque fois de nouvelles libertés d'action (souvent au prix de nouvelles contraintes), des temporalités inouïes, des rapports adaptés à un certain état actuel du monde et des choses.

5 Ces époques, toutes associées à des appareils, se déploient au sein d'une ère nouvelle caractérisée par un matériau inédit de la culture : le numérique. 
6 Avec la photo, par exemple : la différence qui sépare la photo-numérique de la photoargentique n'est pas de degré mais de nature, notamment parce que leurs matières respectives sont radicalement étrangères l'une à l'autre.

7 Alors que la matière de la photo-argentique, faite de sels d'argent et de papier, est chimique, la matière de la photo-numérique est langagière. Elle est faite de signes et de codes informatiques, d'algorithmes. Alors que dans un appareil de photo-argentique la lumière agit sur une surface chimiquement sensible, l'appareil de photo-numérique est, lui, muni de capteurs et de processeurs grâce auxquels l'action de la lumière est convertie en signes informatiques - en langage.

La photo-numérique rompt l'homogénéité de matière entre les choses et les images qui, dans la photo-argentique, était assurée par la lumière et les sels d'argent. Un contact physique a bien lieu entre les choses et le dispositif numérique de saisie, mais il ne s'accompagne plus d'un échange énergétique entre les choses et les images. La transformation ne s'opère plus de chose à chose, des choses du monde à des imageschoses, mais de chose à image-langage.

9 On passe du monde chimique et énergétique des choses et de la lumière au monde logico-langagier des images numériques. L'ancienne continuité matérielle entre la chose et son image argentique est brisée au profit d'une conversion de la matière en langage - autrement dit, au profit d'une virtualisation. C'est donc dès l'étape de la saisie que s'opère la rupture du lien physique et énergétique, rupture qui fonde l'altérité essentielle par laquelle la photo-numérique diffère en nature de la photo-argentique.

10 Cette rupture du lien physique et énergétique entre les choses et les images équivaut à une rupture du régime de l'empreinte institué par la photographie au milieu du XIx siècle.

11 L'ère du numérique sonne la fin de l'époque bénie que Roland Barthes a décrite comme celle où « le référent adhère » aux images photographiques, où chaque scène ou chose figurée «a été » avant de venir s'inscrire et se fixer sous la forme d'une empreinte de temps et d'espace dans la matière précieuse des images d'argent. Époque bénie, donc, où les images figuraient en fixant, en isolant, en sacralisant et, en quelque sorte, en édifiant des monuments iconiques aux choses. Époque bénie, enfin, où cet appareillage (technique) et cet apparat (esthétique) de l'enregistrement par contact des apparences supportaient un puissant régime de vérité.

Dès lors que les enregistrements numériques sont langagiers, la rigidité des images se dissout dans une ductilité infinie. Alors que la retouche était un tabou de la photoargentique, un acte de lèse-vérité, elle est devenue l'état ordinaire de la photonumérique au travers des logiciels de traitement d'images qui sont livrés avec les appareils... En termes deleuzien: la photo-argentique fonctionne sur le mode du «moule» (une forme fixe générant une série d'occurrences identiques) tandis que la photo-numérique ressortit, elle, à la "modulation» - chaque image étant emportée dans les devenirs de ses infinies transformations et variations.

13 En pratique, les images numériques se caractérisent par une perte d'origine, par une dissolution du référent, par une sorte de détachement du monde. Quant aux spectateurs, ils assistent à un devenir-image du monde et à l'avènement d'un tout autre régime de vérité - l'ère numérique devenant l'ère du doute, l'envers de l'époque des illusions de vérité qui était accrochée aux photo-argentiques. Un vrai doute succède ainsi à une fausse certitude de vérité. 
Si le matériau logico-langagier de la photo-numérique brise le lien matériel entre les images et les choses qu'avait noué la photo-argentique, si donc il fait vaciller l'ancien règne de l'empreinte, il est celui par lequel s'édifie l'ère numérique des sociétés d'aujourd'hui. En apparence si peu matériel, ce matériau a pourtant une matérialité, celle d'un langage, qui est assez forte pour ouvrir une nouvelle ère dans la culture et la civilisation, et, accessoirement, pour nous inciter à renoncer à la fausse et trompeuse notion de "dématérialisation » du monde. Aussi ténue tactilement soit-elle, une autre matière n'est pas une absence de matière, mais une version différente de la matière iconique.

L'un des grands ressorts du matériau numérique est d'être commun à tous les types d'images - photo, vidéo, sonores et textuelles - qui peuvent dès lors se combiner et se côtoyer au sein d'un même document, dont la page internet est le parangon. Cette unité de tous les types d'images à l'ère numérique se substitue ainsi -en fonction de nouveaux enjeux - à l'ancienne unité de la chose et de son image scellée sous la forme de l'empreinte à l'ère photographique.

La surface d'inscription ainsi que la vitesse, le mode et l'aire de circulation, sont quelques uns des autres traits caractéristiques et distinctifs des images. À cet égard, l'ère nouvelle du numérique s'impose au détriment de matériaux plus traditionnels tels que le papier qui est, avec d'autres, durement concurrencé dans les domaines de la presse, du livre et des images, y compris des images fixes de la photo.

17 La photo-argentique et le papier appartiennent au même monde thermodynamique. Ils en partagent les protocoles de fabrication, les modes et les rythmes de circulation, la tactilité. Autrement dit, dans l'état du monde d'aujourd'hui, les lenteurs, les rigidités, les coûts, les rapports au monde, etc.

Autant de domaines que les images numériques viennent reconsidérer d'une façon si radicale qu'elles instituent une nouvelle ère : celle du langage pour matériau, celle des écrans pour surface d'inscription, celle des réseaux planétaires pour espace, celle de l'immédiateté pour vitesse, celle de l'interactivité pour mode de réception, celle d'un détachement assumé du monde physique pour régime de vérité, celle d'une unité de matière et d'une profusion pour dresser un monde autonome - sorte de hors-monde de tous les types d'images, celle de la logique implacable d'un langage opératoire dépourvu de sens comme loi cachée de l'apparente souplesse, etc.

19 Autant d'éléments qui sont autant de fractures dans la culture, bouleversant les temps et les espaces, les vitesses, les matières, les regards et les savoirs, et bien sûr les façons de travailler, de créer, et plus généralement de vivre.

\section{RÉSUMÉS}

André Rouillé est un spécialiste de l'histoire de la photographie en France, à laquelle il a consacré un livre publié chez Macula. Depuis cet ouvrage, il se consacre, dans le cadre du site http:// www.paris-art.com/, à la politique culturelle en France et analyse l'effet des modifications technologiques sur l'art photographique. L'irruption de la photo numérique, supplantant 
définitivement la photo analogique, impose de reconsidérer les cadres de l'esthétique dont la photo analogique avait hérités. En fait, c'est le classicisme de cette esthétique, hérité de la Renaissance albertienne, qui succombe du fait de l'écriture numérique qui est totalement soumise à la communication. Les images obtenues par un smartphone sont destinées à être immédiatement diffusées sur des réseaux sociaux comme éléments d'une distinction sociale mouvante et non comme témoignages d'un existant. On passe donc de l'état d'une image qui pourrait me poindre (Barthes) à une fascination pour l'impermanence.

\section{INDEX}

Mots-clés : photographie analogique, numérique, classicisme photographique, cadre, flou, communication 